Service social

\title{
Évaluation d'un programme de responsabilisation de jeunes contrevenants
}

\section{Marie Bruneau, Michel Laflamme et Marie-Josée Linteau}

Volume 35, numéro 1-2, 1986

Recherche - Action - Évaluation

URI : https://id.erudit.org/iderudit/706297ar

DOI : https://doi.org/10.7202/706297ar

Aller au sommaire du numéro

Éditeur(s)

École de service social de l'Université Laval

ISSN

1708-1734 (numérique)

Découvrir la revue

Citer cet article

Bruneau, M., Laflamme, M. \& Linteau, M.-J. (1986). Évaluation d’un programme de responsabilisation de jeunes contrevenants. Service social, 35(1-2), 105-125. https://doi.org/10.7202/706297ar d'utilisation que vous pouvez consulter en ligne.

https://apropos.erudit.org/fr/usagers/politique-dutilisation/ 
BRUneaU, Marie, agente de programmation au Centre de services sociaux de Québec.

LAFLAMME, Michel, consultant en programmation au Centre de services sociaux de Québec.

LINTEAU, Marie-Josée, professionnelle au service de protection et de consultation sociales, Ministère de la santé et des services sociaux (Québec).

\section{Évaluation d'un programme de responsabilisation de jeunes contrevenants}

\section{Marie Bruneau Michel Laflamme Marie-Josée Linteau}

La mise en application de la Loi sur les jeunes contrevenants, en 1984, a insufflé des perspectives nouvelles dans l'action des professionnels. Ceux-ci étaient conviés à développer une approche éducative centrée sur la responsabilisation, impliquant à la fois le jeune, sa famille et la communauté. Mais déjà quelques années avant l'entrée en vigueur de cette loi, des intervenants du Centre de services sociaux de Québec (C.S.S.Q.) appliquaient un programme visant ce même but et favorisant le renouement social du contrevenant avec sa collectivité, dont il s'était trouvé coupé par la commission d'un délit.

Depuis la mise en vigueur de la loi, aucune évaluation systématique de ce type de programme de responsabilisation n'a été réalisée au Québec. Aussi, un groupe d'intervenants du C.S.S.Q. a mis sur pied un projet de recherche ${ }^{1}$ subventionné dont le but principal était de mesurer l'impact sur le jeune contrevenant d'un tel programme incluant diverses mesures de responsabilisation.

Le présent article se limitera d'abord à présenter les fondements du programme, en identifiant les différentes tendances dans la conception du problème de la délinquance. Ensuite, les principales questions à 
l'étude concernant l'évaluation de la responsabilisation seront sommairement rapportées, avant d'en arriver à la discussion du concept même et à la présentation de propositions pour la mesurer chez de jeunes contrevenants. Enfin, nous présenterons la population à l'étude, les instruments de mesure employés et le devis méthodologique de recherche.

Une fois cette recherche en cours terminée, c'est la pratique sociale qui devrait, au premier chef, bénéficier de ses résultats. En effet, les orientations de l'intervention en délinquance s'en trouveront alors validées ou remises en question ou encore apparaîtront comme rentables dans un contexte particularisé et pour une clientèle particulière.

\section{Les fondements du programme}

Durant les cinq premières années d'application de la Loi québécoise sur la protection de la jeunesse (1979), plusieurs situations de jeunes contrevenants ont été traitées par la voie d'un processus parajudiciaire (déjudiciarisation ou «diversion » juvénile) en postulant que ces adolescents étaient capables d'assumer activement leurs responsabilités face aux infractions commises. L'esprit de cette loi de 1979 favorisait également l'implication des acteurs gravitant autour du jeune afin d'atteindre l'objectif de leur prise en charge par leur communauté.

À la même période, de nombreuses initiatives visant des objectifs de responsabilisation individuelle et communautaire étaient tentées dans les centres de services sociaux du Québec, mais ces tentatives ont été trop dispersées pour qu'on puisse ressortir un cadre uniforme d'intervention et porter un jugement évaluatif d'ensemble. Conséquemment, la pratique a oscillé toujours un peu au gré des intervenants, des valeurs véhiculées à partir de divers courants et philosophies pouvant parfois se confronter.

La Loi fédérale sur les jeunes contrevenants, sanctionnée en 1982 mais proclamée en 1984 seulement, mit en ouvre ${ }^{2}$ une conception fondée sur les droits et les responsabilités des jeunes qui ont des démêlés avec la justice. D'une part, elle met l'accent sur l'idée que les adolescents doivent être tenus responsables de leur conduite illicite et que la société a le droit de se protéger contre une telle conduite. D'autre part, elle reconnaît que les adolescents ont des besoins spéciaux et qu'ils ne sauraient être assimilés aux adultes quant à leur degré de responsabilité et aux conséquences de leurs actions, vu leur état de dépendance et leur niveau de développement et de maturité. 
Dans la pratique traditionnelle issue de l'ancienne loi fédérale sur les jeunes délinquants, l'emphase de l'intervention était mise sur l'action individuelle et familiale, dans une perspective de traitement qui risquait d'accentuer la dépendance du jeune, d'isoler la famille avec son problème et de négliger les facteurs socioculturels dans l'explication et le traitement de la délinquance. L'approche préconisée par le programme sous évaluation a davantage une portée éducative centrée sur l'apprentissage de la responsabilisation individuelle et communautaire tel que souhaité par l'esprit de la nouvelle loi fédérale sur les jeunes contrevenants. On pourrait illustrer l'originalité de l'approche proposée par ce programme par le schéma suivant:

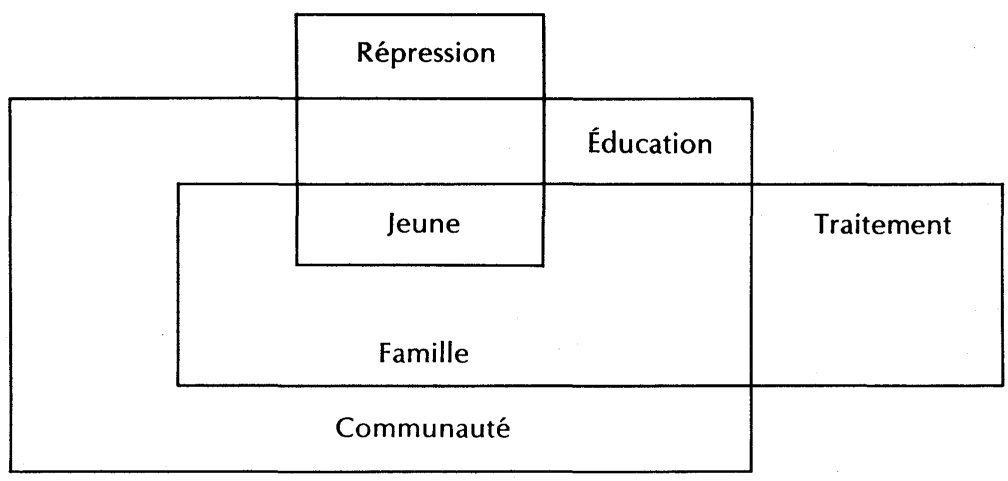

Entre les deux extrêmes, constitués par une intervention de répression auprès du jeune et par une intervention thérapeutique axée sur le jeune et sa famille, on peut identifier la perspective du programme qui, tel que précisé plus haut, en est une d'éducation qui vise l'apprentissage de la responsabilisation chez le jeune, dans sa famille et dans sa communauté, mais qui n'exclut pas, parallèlement, l'action thérapeutique. Sans renier un certain pouvoir - qu'il prenne une couleur tantôt répressive ou tantôt thérapeutique - tant au niveau des décisions à prendre que des moyens pour les actualiser, les tenants de cette approche (Bruneau et Linteau, 1983) ${ }^{3}$ se dissocient de tout monopole du pouvoir qui risquerait, à ce niveau, d'encourager une dépendance passive du jeune. L'approche préconisée donne plutôt à l'intervention un sens systématiquement collectif. Ceci implique qu'elle doit favoriser le retour de l'individu au lien d'origine avec sa communauté, lien qui demeure le seul instrument valable d'un 
enrichissement individuel et collectif. On pourrait parler, en ce sens, d'une sorte de renouement social.

La philosophie d'intervention qui anime les rapports avec le jeune dans l'actualisation de ce programme situe le délit en référence au milieu dans lequel le jeune est inscrit et évolue. Autant il est inscrit dans un milieu et dans un contexte, autant le délit commis doit-il s'inscrire lui aussi dans cette même perspective; on ne peut alors dissocier ou évacuer le délit du milieu ou du contexte dans lequel il est commis. Au plan de l'intervention, la tendance qu'ont des communautés à s'organiser pour se protéger contre leurs jeunes délinquants n'est pas encouragée. Au contraire, l'objectif est plutôt de créer un esprit communautaire qui apprivoise la peur suscitée par la délinquance et qui intègre ensuite positivement cette dynamique qui, auparavant, confrontait la communauté.

La vision de la problématique permet d'identifier l'acte délinquant comme relié à une crise, en marge de laquelle une coupure est consacrée entre le jeune et la communauté dont il transgresse les règles ou dont il se dissocie. Dans une perspective de renouement social, l'état de crise est utilisé comme moteur d'intégration sociale selon un processus au cours duquel la réaction parentale devient révélatrice de valeurs, de préoccupations, et mobilisatrice des ressources, en même temps que le délit devient occasion d'émergence de solutions alternatives ou de comportements nouveaux et responsables.

Dans cette conception, le client n'est pas seulement un utilisateur de services; mais plutôt, et dans l'esprit qui a animé la Commission Charbonneau ${ }^{4}$, il est appelé à être un élément actif dans la recherche de sa reconnaissance sociale. Le praticien joue son rôle d'intervenant, mais son pouvoir est tributaire de celui des autres intervenants de la communauté qu'il mobilise.

En ce qui concerne le jeune, les objectifs ont trait à la conscientisation, à la responsabilisation, à la restauration de l'estime de soi et au support à la participation sociale. Face à la communauté, on vise aussi la conscientisation et la responsabilisation, mais on y greffe en plus son implication active auprès de ses jeunes marginaux. Ces objectifs s'opérationnalisent dans le cadre d'interventions de groupe pour les parents et les jeunes, d'entrevues individuelles ou familiales, selon les composantes de la situation, et par des contacts constants auprès d'autres entités de la communauté (groupes sociaux, groupes de loisirs, conseils de ville, etc.). 


\section{Évaluation de la responsabilisation : questions à l'étude}

La question fondamentale de notre recherche a trait à l'efficacité des interventions de responsabilisation réalisées auprès des jeunes qui ont commis des délits, et notre hypothèse est la suivante :

"L'application à de jeunes délinquants d'un programme de responsabilisation personnelle axé sur une perspective communautaire améliorera leurs attitudes et leurs comportements face à l'exercice de la responsabilité.»

En raison de cette hypothèse, nous sommes d'abord amenés à vérifier si l'application du programme produit des comportements responsables chez les jeunes qui y sont soumis. Ces comportements réfèrent d'abord à la réalisation, par les jeunes, de mesures réparatrices ou compensatrices consécutives au délit commis; le fait de s'abstenir de commettre d'autres délits, dans les mois qui suivent, est également considéré comme un comportement responsable.

On cherche ensuite à mesurer s'il y a un changement d'attitudes chez les jeunes, changement manifesté dans leurs performances aux divers instruments de mesures retenus à cette fin. Un critère important touche l'application même du programme : s'assurer qu'il a comporté une perspective communautaire dans les mesures concrètes de responsabilisation utilisées avec les bénéficiaires dudit programme d'intervention.

On se demande également s'il produit un impact différent selon qu'il est appliqué à différentes catégories de délinquants (conformistes, intermédiaires ou persistants); cette question repose sur la soushypothèse qu'un impact plus significatif devrait être observé chez les jeunes qualifiés de délinquants intermédiaires.

Une série d'autres questions feront aussi l'objet d'analyse ou de vérification; ainsi :

- est-ce que le fait d'utiliser plus d'une mesure de responsabilisation a une incidence plus grande sur le jeune et, si tel est le cas, quelle combinaison de mesures produit un effet plus grand ?

- est-ce que l'application des mesures produit un profil unique d'amélioration des attitudes ou si, au contraire, elle a un impact sur certains secteurs de performance très différenciés?

- quel type de mesures (conciliation, travaux communautaires, probation, etc.) amène le plus de changements aux résultats manifestés dans les échelles? Et ceci, pour chacune des typologies et pour chaque tranche d'âge? 
—à l'aide d'un indice synthétique regroupant plusieurs questions, par exemple sur la participation du jeune à la détermination de la mesure de responsabilisation, peut-on faire un lien entre les résultats du programme et le niveau de conscientisation ou d'implication du jeune dans l'intervention?

- peut-on observer un lien entre les résultats du programme et une intégration ou une projection positive et active du jeune? L'indice synthétique développé à cet effet regroupe plusieurs sous-questions relatives au contrôle parental, au désengagement scolaire du jeune, à son type de loisirs, à l'entretien de rapports positifs avec l'autorité, à la présence chez lui de projets à court ou long terme, etc.

\section{Le concept de responsabilisation}

Dès le départ, il faut souligner la carence de littérature permettant de cerner la définition de la responsabilisation, ce qui constitue une certaine limite et oblige à une approche périphérique de cette notion. Néologisme qui s'est davantage développé dans le domaine de l'éducation, de la justice et des disciplines d'aide, ce mot a, dans le sens commun, la signification de "rendre responsable». On doit donc référer à une définition de la responsabilité pour appréhender le concept de responsabilisation.

Le dictionnaire Larousse définit la responsabilité comme l'obligation de réparer une faute ou de remplir un engagement. Sous l'aspect civil, elle devient l'obligation de réparer les dommages causés à autrui et, sous l'angle pénal, elle devient l'obligation de supporter le châtiment prévu pour l'infraction commise.

La responsabilisation, comme tous les mots qui ont cette terminaison, implique une idée de mouvement et, dans ce cas-ci, le mouvement de rendre responsable. La responsabilisation sera donc un processus d'action visant à rendre responsable une personne qui ne l'est pas, qui a omis de l'être circonstanciellement ou qui tarde à le devenir de façon active.

On ne peut, conséquemment, séparer ce concept de celui d'apprentissage, car la personne deviendra responsable ou apprendra à être responsable dans la mesure où elle aura des occasions de réparer ses fautes, de corriger ses erreurs ou de remplir ses engagements.

Quand l'entraînement à un comportement responsable n'est pas adéquat ou n'existe pas, par défaut des parents, des agents d'éducation ou des structures sociales, il s'ensuit une insuffisance normative 
conduisant à des comportements dérogatoires parfois en contravention avec la loi.

Dans ce type de situation qui confronte un jeune pour les premières fois, une approche éducative apparaît indiquée. En effet, si on apprend au jeune à comprendre le tort causé par son acte et à concevoir qu'il doit en assumer (ou apprendre à en assumer) les conséquences tout en lui fournissant l'occasion de s'engager à réparer le tort causé, il modifiera probablement son schéma comportemental pour le rendre plus conforme aux exigences de la vie en société. Ainsi, le jeune apprendra à se reconnaître comme capable de remplir ses engagements.

Discutons également d'une divergence à ce point de vue qui est véhiculée par les tenants d'une approche plus répressive à l'égard des délinquants. Ces opposants, qui reflètent d'ailleurs une certaine tendance sociale, voudraient voir les jeunes payer le plein prix pour leurs actes dérogatoires et assumer, au même titre que les criminels adultes, les conséquences pleines et entières de leurs actes. On pourrait retrouver le fondement de cette tendance dans le partage d'une option philosophique et juridique selon laquelle la reconnaissance du libre arbitre rend l'homme capable de choisir et, en même temps, responsable de supporter les conséquences de ses choix. C'est d'ailleurs là un des principaux principes sur lesquels s'est fondée l'École classique de droit pénal au XVIIII siècle (Szabo, 1970). ${ }^{5}$ Cette vision des choses tient à une image classique de l'homme, maître parfait de sa destinée, de ses instincts et de ses actions. Dans cette École, on reconnaît à l'homme la liberté, donc la responsabilité personnelle totale des actes individuels.

À cette tendance s'est opposée celle véhiculée par ce qu'on a appelé l'École positiviste du XIXe siècle (Szabo, 1970$)^{6}$, qui veut prendre en compte les influences psycho-socio-culturelles qui façonnent la personnalité et qui relativisent la liberté et donc la totalité de responsabilité personnelle. En s'inspirant de cette perspective, on proposait de substituer au principe de la responsabilité personnelle un principe de responsabilité sociale comme base de l'action de la société à l'égard de ses criminels.

Certains criminels étant reconnus récupérables grâce à un traitement approprié, il s'est développé une législation basée sur l'individualisation de la peine, celle-ci permettant alors la rééducation du criminel et sa réconciliation avec la communauté ou avec les normes de la vie en société. Les travaux communautaires, imposés aux adultes comme alternative à l'emprisonnement, sont un exemple de mesure individualisée favorisant la réadaptation sociale et comportant un 
aspect de rééducation. En somme, ces principes de pénologie appliqués au monde des adultes se sont transposés au domaine de la délinquance juvénile et on a voulu avoir aussi, à l'égard des jeunes une action dissuasive, une opération de contrôle et une intervention éducative sinon de rééducation.

L'action menée à l'égard des jeunes délinquants s'inspire du principe de responsabilité sociale, certes, mais aussi de celui de responsabilité personnelle qui conduit à imposer une conséquence à l'acte répréhensible commis à l'encontre d'intérêts d'individus ou de la société. Dans ce domaine, les approches pour appréhender la responsabilité tireront leurs assises, autant sinon plus, du domaine de l'éducation et de la psychologie que de la criminologie et du droit.

Dans cette perspective, on peut avancer qu'il est de la responsabilité comme de la liberté : elle n'est pas acquise en une seule fois, mais on doit plutôt en faire un apprentissage gradué. Glasser ${ }^{7}$ affirme, à ce propos, qu'il ne suffit pas d'affirmer la responsabilité d'une personne (surtout quand il s'agit d'un jeune en développement) et de la déclarer responsable des conséquences de ses actes, mais qu'il faut aussi l'aider à devenir responsable. Pour les tenants de cette approche, la responsabilisation s'opère, notamment, par le refus de comportements irrationnels comme modes d'adaptation au monde, par la confrontation avec la réalité, par l'enseignement de comportements responsables et satisfaisant les besoins du jeune et par la reconstruction de l'estime de soi.

Sur un plan développemental, les mesures responsabilisantes auront un impact sur le comportement du jeune si celui-ci en vient à reconnaître ce qu'il a fait et sa responsabilité conséquente (dans le sens qu'il prend conscience du tort causé, qu'il accepte d'en assumer des conséquences et qu'il prend un engagement formel à réparer ou compenser le tort) et à admettre que des possibilités alternatives existent pour régler son problème ou exprimer ce que son délit ou son acte voulait exprimer (élargissement du répertoire comportemental) (Malcuit, Granger et Larocque, 1972) ${ }^{8}$.

Nous avons vu, plus haut, que l'apprentissage de la responsabilité (responsabilisation) est possible, chez le jeune, dans la mesure où il peut s'engager à réparer ou compenser le tort causé et être reconnu comme capable de remplir ses engagements. On peut penser que cette reconnaissance extérieure de sa capacité à se responsabiliser aura une incidence positive sur son estime de soi et que l'intériorisation de ce sentiment constituera un des contrôles internes (auto-contraintes) (Reckless, 1961) ${ }^{9}$ devant l'aider à maintenir un comportement responsable. 
Et quand on sait que l'estime de soi des délinquants est généralement faible (Leahey, 1974 10; Jensen, 197211), il devient plus important de travailler à la reconstruire et de chercher à éviter les interventions qui risquent de l'affaiblir davantage, ce qui serait le cas d'un étiquetage issu de l'utilisation inappropriée du processus judiciaire (Morris, 1978). ${ }^{12}$

Pour que la responsabilisation ait un impact sur la réduction de la délinquance comme comportement individuel et comme phénomène sur le territoire concerné ${ }^{13}$, il faut voir se réaliser certaines actions ou certains mouvements chez le jeune, ses parents, l'intervenant et la communauté. Fidèles à la définition précédente du concept de responsabilisation et à l'illustration opérationnelle qui en sera faite dans les instruments de cueillette de données, voyons ci-après certaines de ces actions ou de ces mouvements.

\section{Le jeune}

Globalement, il faut arriver à ce qu'il pose un geste ponctuel de réparation du tort causé et acquière pédagogiquement un comportement responsable devant éviter la récidive. Il pourra y avoir responsabilisation s'il réalise certaines des activités suivantes: conciliation avec la victime (remboursement, travail compensatoire ou réparatoire, lettre d'excuse, etc.), travail communautaire, activités de réflexion (rencontres de groupe, réflexion dirigée à partir d'un texte, etc.), dons à des organismes communautaires, mise sous probation (en milieu naturel) et mise sous garde (en milieu institutionnel). Il y aura également responsabilisation s'il progresse dans l'acquisition des attitudes et comportements révélateurs d'une intégration sociale: création d'un lien avec la communauté, apprentissage de normes ou de règles sociales, compréhension et regret du tort causé, amélioration d'aptitudes sociales, arrêt du comportement délinquant et autres acquisitions dans le processus de responsabilisation tel que mesuré par les différents tests utilisés.

\section{Les parents}

Globalement, les gestes de responsabilité seront assimilables à la réaction familiale adéquate dont il est question plus haut. Les parents responsables ou responsabilisés sont réputés tels s'ils : contrôlent le comportement et les sorties du jeune, supervisent occasionnellement des mesures réparatrices, recréent avec lui un lien fonctionnel, enseignent des comportements responsables et s'impliquent activement dans une rencontre de groupe consécutive au délit et au signalement. 


\section{L'intervenant}

Globalement, l'intervenant veut responsabiliser le jeune, ses parents et la communauté, mais il doit lui-même témoigner, dans son action, de gestes qui y correspondent; on reconnaîtra une telle perspective s'il : fournit au jeune des occasions de réparer ses fautes ou ses erreurs, le reconnaît comme capable de s'engager et de remplir ses engagements, refuse ses comportements irrationnels comme modes d'adaptation au monde, le confronte avec la réalité, lui enseigne des comportements responsables (c'est-à-dire satisfaisant ses besoins tout en étant socialement acceptables) et l'aide à reconstruire son estime de soi. L'application adéquate du programme nécessite de telles attitudes chez l'intervenant, mais ce point ne sera pas vérifié dans le cadre de la présente recherche.

\section{La communauté}

Globalement, la communauté responsable ou responsabilisée pose des gestes visant l'intégration des jeunes comme groupe si elle leur donne des occasions suffisantes de se retrouver en milieux d'appartenance et de se réaliser comme personnes à travers des projets et à travers leur participation à la vie communautaire. Dans une démarche de responsabilisation, la communauté, à travers ses représentants: identifie des projets communautaires d'aide à la jeunesse, investit du temps ou de l'argent pour la mise en place de projets ou de ressources, crée et maintient des ressources en fonction des besoins locaux des jeunes, les fait participer à cette démarche de mobilisation ou d'organisation, fournit et supervise des occasions de travail communautaire compensatoire. L'impact communautaire qui accompagne ce programme d'intervention intégrée ne sera pas objet d'évaluation ici en raison de l'ampleur de l'instrumentation nécessaire ; cette vérification pourrait faire, à elle seule, l'objet d'un autre projet de recherche.

\section{Mesure de la responsabilisation}

À partir de l'étude du concept de responsabilisation tel que présenté antérieurement, deux indices, que nous décrirons maintenant, permettront de mesurer cette variable. 


\section{La présence d'un geste factuel de responsabilisation}

William Glasser ${ }^{14}$ définit la responsabilité comme l'aptitude à satisfaire ses besoins et à le faire d'une façon qui ne prive pas les autres de leurs aptitudes à satisfaire les leurs. Il affirme aussi qu'il faut aider l'individu à devenir responsable par le refus de comportements irrationnels comme mode d'adaptation au monde et par la confrontation avec la réalité.

De son côté, Cusson ${ }^{15}$ rappelle que la cohérence sociale exige qu'un blâme sanctionne le délit, mais que la nécessaire reconstruction de l'estime de soi passe par l'enseignement de comportements responsables satisfaisant les besoins (conséquences de l'acte liées aux besoins et capacités du jeune). Cette idée d'un geste factuel de responsabilisation a généré la sous-hypothèse selon laquelle les jeunes dont la mesure (conséquence de l'acte) comporte une activité formelle axée sur la réparation ou la compensation feront un apprentissage les amenant à devenir des personnes plus responsables.

\section{La portée éducative de la mesure}

Une première sous-hypothèse nous amène ici à croire que, si la mesure de réparation a été choisie en relation directe avec les capacités de réalisation et les besoins de conscientisation et d'apprentissage du jeune, et si ce dernier a participé activement à la détermination de ladite mesure, celle-ci a une portée éducative et lui permet d'augmenter son niveau de responsabilisation.

Une deuxième sous-hypothèse, en regard de la portée éducative de la mesure, veut que le niveau de responsabilisation augmente quand il existe un lien de contingence direct entre le délit (nature et gravité) et le geste de réparation choisi. Ce lien est plus direct lorsque la mesure s'accomplit d'abord auprès de la victime et auprès de la communauté immédiate du jeune, ensuite auprès de la communauté éloignée et, enfin, lorsqu'il y a mise sous garde.

La confrontation avec la réalité aura un meilleur impact si on rend le jeune conscient qu'on ne travaille pas sur sa "personnalité irresponsable" mais plutôt sur un manque de responsabilité dans un comportement précis ou lors de la commission d'un acte répréhensible identifiable.

Pour qu'il puisse établir un lien entre son comportement et la conséquence de son geste, il faut d'abord qu'il y ait contiguïté, c'est-àdire la presque immédiateté temporelle entre l'acte et la conséquence. Ce lien réclame également la contingence, c'est-à-dire un lien direct 
entre l'acte reproché et la punition. Si l'individu puni ne constate pas ce lien, la punition n'aura pas la valeur éducative ou thérapeutique escomptée et sera davantage considérée comme une attitude vengeresse du punisseur ou comme une peine qui efface tout (même le manque de responsabilité ayant présidé à la commission de l'acte illégal ou indésirable).

L'extinction du comportement indésirable devra donc s'accompagner d'une sorte d'enseignement correspondant au besoin d'apprentissage du jeune, eu égard à des comportements alternatifs acceptables par l'environnement et susceptibles également de répondre à ses besoins d'affirmation ou d'expression. Un autre facteur prédictif du succès ou de l'efficacité de la punition est la reconnaissance claire d'indices annonçant cette même punition (Laflamme, $1983^{16}$; Malcuit et al., $1972^{17)}$.

Glasser ${ }^{18}$ ajoute également qu'il faut expliquer attentivement aux jeunes les règles ou les normes auxquelles doit se conformer leur comportement, l'expérience démontrant qu'on ne doit jamais prendre pour acquis qu'ils connaissent déjà les paramètres devant régler leur conduite en toute circonstance. II faut se rappeler qu'ils sont en période d'apprentissage et de développement et que leur comportement erratique ou oppositionnel est parfois une recherche de normes sociales asssez importantes pour justifier un rappel, une punition ou une conséquence en cas de transgression.

On a vu plus haut que la portée éducative des mesures constitue un élément essentiel des actes de responsabilisation et repose notamment sur un renouement social du jeune avec son environnement communautaire. On pourrait se demander pourquoi on travaille à établir ou rétablir un tel lien.

Citant Hirshi, qui s'est intéressé au lien en matière de délinquance, Cusson ${ }^{19}$ rapporte que :

"Les adolescents commettent des actes délinquants parce que leur lien à la société s'est affaibli ou rompu, rendant inopérante l'action régulatrice du groupe social. Ce lien individu-société serait constitué de quatre éléments: 1) l'attachement à autrui qui incite l'adolescent à se conformer aux attentes légitimes de l'autre pour ne pas le heurter ; 2) l'engagement dans des activités honnêtes qui le motive à éviter de commettre des délits qui, par les réactions qu'ils risqueraient de provoquer, pourraient nuire à ses projets ; 3) l'implication, qui est simplement le fait d'être occupé pendant ses loisirs ; 4) la croyance ou la conviction qu'on doit obéir aux lois."

Cusson explicite la théorie d'Hirshi en la complétant par une référence à l'étude sociologique de Durkheim; ce dernier ajoute au 
concept de "régulation sociale par l'attachement à l'environnement social» l'idée qu'il est important, pour cet environnement, de manifester une croyance aux valeurs qu'il juge essentielles et d'y être fidèle. Les deux grandes notions régulatrices du comportement social pourraient ainsi être l'intégration au groupe et le blâme issu de l'environnement.

Cusson ${ }^{20}$ renforce cette affirmation en citant Tittle : "Les sanctions qui viennent de nos proches et des membres de notre communauté sont beaucoup plus efficaces pour enrayer le crime que celles que brandit l'État." Malgré l'importance de cette sanction sociocommunautaire, la réhabilitation autant que la rééducation nécessitent des relations franches et même chaleureuses entre le délinquant et les membres de son milieu.

Comme le rapporte Louise Blain-Lambert $(1975)^{21}$ au sujet de jeunes ayant séjourné en institution :

"Il semble que pour réussir pleinement, la réhabilitation doive répondre à trois besoins essentiels du jeune au sortir de l'institution ": que la famille et le voisinage considèrent en lui son intégrité reconquise, sa valeur propre; que l'Église et la Société lui donnent accès au rôle qui revient de droit à tout citoyen intègre (Mailloux, 1974b; voir Bastien, 1975). Pour ce faire, il faut que les membres de la communauté changent leur perception vis-à-vis l'avenir du délinquant et qu'ils participent de façon tangible à la réhabilitation (Kupperstein, 1971 ; Mailloux, 1974a, 1974b ; quatrième congrès des Nations Unies..., 1972; Tanguay, 1974).

Tout en poursuivant l'objectif de protection de la société qui pourrait avoir comme conséquence ultime le retrait du jeune de sa communauté, il ne faut pas négliger la réadaptation personnelle que doit vivre le jeune. Dans la recherche de sa reconnaissance sociale, son questionnement est facilité par la mise en contact directe du jeune avec sa victime (sa communauté) à qui il a causé préjudice.

\section{Population de l'étude d'évaluation}

La population est formée de toute la clientèle du programme d'intervention et comprend les jeunes âgés de 12 à 18 ans référés au Substitut du procureur général en raison de la commission d'une infraction aux lois fédérales pendant la période du $1^{\text {er }}$ avril 1984 au

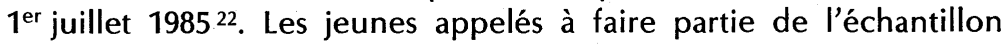
devaient toutefois provenir des vingt et une municipalités du territoire précédemment identifié. Ces municipalités ont été choisies à partir de critères tels la représentation urbaine et rurale, le volume de situations 
référées et la collaboration offerte au projet de recherche par les intervenants affectés à ces territoires.

Après l'application des mesures de responsabilisation, la population à l'étude fut classifiée en trois sous-catégories: les délinquants persistants, les délinquants intermédiaires et les délinquants conformistes. Cette catégorisation est importante, car l'application du programme à l'ensemble des jeunes contrevenants veut éclairer les praticiens sur le lien pouvant exister entre l'appartenance à un type de délinquance et le bénéfice que le jeune peut retirer de mesures de responsabilisation.

Cette typologie trouve ses fondements dans une recherche de Marcel Fréchette ${ }^{23}$ intitulée "Portrait de la délinquance ", et qui visait à étudier l'agir délictueux de l'adolescent pris en charge par le Tribunal des mineurs. Après avoir rigoureusement défini la délinquance telle qu'étudiée dans sa recherche, Fréchette passe en revue les diverses manifestations de l'agir délictueux selon des critères fixes (nombre moyen d'actes, distribution de fréquences des sujets, modèles dominants dans le modus operandi, etc.) et en arrive à la construction d'un instrument bâti strictement avec des données sur le comportement délinquant qui partage les sujets selon le degré d'intensité ou de gravité de leur délinquance. Cet instrument, appelé "Échelle d'intensité de la délinquance", s'avère donc capable de mesurer, selon un mode quantitatif, la force de l'engagement dans l'agir antisocial. Le principe de base qui sous-tend pareille mesure est celui de la convergence d'un nombre optimal d'indicateurs ou d'indices, chacun de ceux-ci représentant une dimension importante du phénomène et reposant entièrement sur l'observation soit des actes eux-mêmes, soit des modes de passage à l'acte, et qui s'expriment ainsi d'une façon strictement comportementale et concrète.

À partir du tableau qui suit, on peut noter les indices choisis pour bâtir cet instrument et utilisés pour répartir les sujets de la recherche selon l'une ou l'autre des sous-catégories.

Au moment de l'élaboration de notre devis de recherche, nous avions énoncé une fréquence théorique pour répartir les sujets dans les sous-catégories selon ce qui devait normalement apparaître en se fondant sur les travaux de Fréchette ou sur l'expérience acquise par les professionnels en délinquance. Au moment où notre recherche s'achève, on peut noter que les 239 sujets de l'échantillon se répartissent selon les proportions escomptées, soit $59 \%$ (141) de délinquants conformistes, $24 \%$ (57) de délinquants intermédiaires et $17 \%$ (41) de délinquants persistants. 


\section{Typologie des délinquants}

\section{Les délinquants conformistes}

- Nature du ou des délits

- vol à l'étalage

- vol avec effraction

- vol simple

- dommages et méfaits

- infractions aux lois sur les stupéfiants

Les délits sont mineurs et occasionnels et sont exempts de violence contre les personnes.

- Nombre de délits

On peut parler ici de quelques délits (un à cinq).

- Caractéristiques du "modus operandi"

Les actes sont souvent commis sous l'effet et l'entraînement d'une autre personne, spontanément, de manière irréfléchie. Ils sont motivés par un désir de jeu et d'excitation (ignorance, défi, incitation).

\section{Les délinquants intermédiaires}

- Nature du ou des délits

Mêmes que pour les délinquants conformistes.

- Nombre de délits

Le nombre de délits peut être plus important que chez les conformistes (un à dix) en autant qu'ils ne revêtent pas un caractère marqué de gravité objective. Quel que soit leur nombre, les délits doivent constituer une (des) récidive(s), les mesures antérieures n'ayant pas apporté les résultats escomptés.

- Caractéristiques du "modus operandi »

La planification des délits a exigé une préméditation. La perte de contrôle est aussi présente dans la réalisation de l'acte, ce qui risque de rendre le préjudice à la victime plus important. La planification a un caractère utilitaire.

\section{Les délinquants persistants}

\section{- Nature du ou des délits}

Les agirs sont plus graves et s'aggravent encore, généralement. Ils comportent, entre autres, des vols ainsi que des attaques sur la personne ainsi que d'autres délits qui font croire à une violence installée.

\section{- Nombre de délits}

Il y a persistance des gestes délictuels dans le temps, un nombre élevé de délits (cinq et plus), dont certains graves. Ce qui les distingue de la catégorie précédente c'est l'importance du nombre d'antécédents et l'échec confirmé des mesures antérieures. On constate une courbe ascendante dans les comportements délictueux allant de l'acte bénin à l'acte très sérieux.

- Caractéristiques du "modus operandi "

Délits planifiés, spectaculaires, à l'intérieur d'un gang ou à la solde d'un réseau. Une circonstance aggravante est la planification avec une pleine conscience. 


\section{Les échelles de mesure}

La mesure du degré de responsabilisation des adolescents contrevenants a nécessité l'utilisation de quatre échelles déjà validées et utilisées dans un grand nombre d'études criminologiques, en plus de l'emploi de deux échelles créées à même la présente recherche et destinées à recueillir des données relatives au vécu social des jeunes et à leur comportement délinquant.

- l'échelle responsabilité (RE) ${ }^{24}$ provient du test "Inventaire de personnalité de la Californie" (C.P.I.). Elle nous donne une cote brute mesurant la responsabilité chez le jeune, c'est-à-dire ses dispositions à être consciencieux, responsable et fiable ;

- l'échelle socialisation (SO) ${ }^{25}$ est également issue du C.P.I. Elle nous donne une cote brute qui nous permet de mesurer le niveau d'apprentissage des rôles chez le jeune et sa maturité sociale;

- l'échelle d'anxiété provient du test IPAT ${ }^{26}$. Elle nous donne une cote brute permettant de mesurer l'anxiété latente et inconsciente chez le jeune et sa capacité de contrôle des tensions ;

- l'inventaire Jesness ${ }^{27}$ contient onze échelles dont nous retiendrons les cotes brutes. Elles donnent un diagnostic différentiel en discriminant entre délinquant et non-délinquant et permettent d'élaborer un portrait clinique ;

- l'échelle $D V^{28}$ (données de vie) systématise différentes données descriptives conduisant à faire le portrait social du jeune. Construite à partir des vecteurs de socialisation vécus par le jeune, cette échelle n'est pas encore validée et son emploi ici en tient compte;

- l'échelle $D F^{29}$ (données factuelles) est constituée de données descriptives visant, cette fois, à cerner le sujet dans ses manifestations délinquantes et à connaître la nature de l'intervention qui a été appliquée à son endroit. Comme la précédente, son utilisation n'est qu'expérimentale.

\section{Le devis de l'étude}

Le devis prévoit une mesure longitudinale et s'applique aux trois groupes définis précédemment : les persistants, les intermédiaires et les conformistes.

Le schéma ${ }^{30}$ suivant permet de saisir le cheminement d'un cas dans le processus de recherche: 


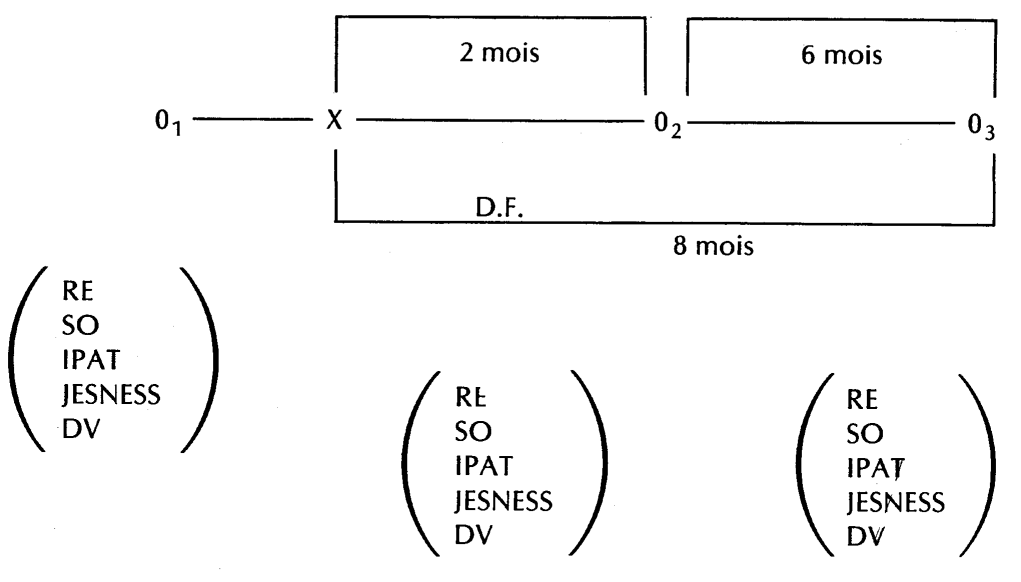

Dès qu'un adolescent est référé au Substitut du procureur général, il est soumis, avant que ne débute l'intervention, à une batterie de cinq tests administrés lors d'une séance individuelle qui dure environ 90 minutes. Cette séance de mesure est appelée $0_{1}$ et les tests administrés sont les suivants :

- sous-test RE (responsabilité)

- sous-test SO (socialisation)

- IPAT (échelle d'anxiété latente)

- Jesness (disposition à la délinquance)

- DV (données de vie).

Dans les jours qui suivent, l'intervention " $X$ " commence ; sa durée est variable et s'étend généralement sur une période de un à six mois, dépendant principalement de la gravité de la situation du jeune. D'autres délais peuvent venir s'ajouter sur lesquels aucun contrôle n'est possible parce que rattachés à l'intervention elle-même. Lorsqu'elle est complétée, le praticien en charge remplit un questionnaire contenant des données factuelles sur la configuration délinquante du jeune et sur l'application de la mesure DF. Deux mois après, le jeune est rencontré pour une deuxième séance de testing $\left(0_{2}\right)$ où la même batterie de tests est administrée et, six mois après cette seconde séance, il est soumis à la même batterie de tests $\left(0_{3}\right)$ pour une troisième fois.

La mesure $\mathrm{O}_{2}$ identifie le sens que prend éventuellement le changement dans les acquis ou dans la configuration de responsabilité chez le jeune. La mesure $0_{3}$ permet d'analyser la persistance de l'effet du programme tel qu'observé lors de la mesure $0_{2}$. Il a été également 
prévu de faire une comparaison des mesures $0_{3}$ des différents groupes de jeunes soumis au programme afin de voir si la persistance dans les acquis (effets du programme) conserve une courbe identique.

\section{Conclusion}

Cette recherche ${ }^{31}$ représente un projet d'envergure, et son plus grand défi est probablement de ne pas rester une démarche théorique mais d'éclairer plutôt la pratique quotidienne des intervenants en délinquance juvénile. C'est la première étude systématique sur un pareil type de programme, et, parce qu'elle est originale, elle comporte divers avantages et désavantages.

Il est par exemple avantageux d'avoir le champ largement ouvert, d'avoir l'impression de défricher un secteur neuf de connaissances sur la pratique, de travailler à l'émergence d'un devis de recherche créateur, etc. Mais ces avantages sont fragiles et se transforment facilement en inconvénients et même en risques. En effet, l'insuffisance ou l'absence de modèles de référence préalables oblige à des remises en question nombreuses et à des exercices de simulation répétés afin de garantir la validité du devis, la pertinence du plan d'analyse, la fiabilité des interprétations et la sûreté des assises sous-tendant les conclusions.

Une autre difficulté a trait aux limites de la littérature disponible sur un concept relativement nouveau et jusqu'ici peu ou mal cerné. Par exemple, le concept de responsabilisation est central dans cette étude et peu d'auteurs ou de chercheurs se sont attachés à le rendre opératoire. Nous avons donc eu à en proposer une compréhension opérationnelle rendue nécessaire par l'importance que représente la responsabilisation dans l'actualisation de la Loi fédérale sur les jeunes contrevenants.

Dans le cadre d'une recherche voulue utile pour la pratique sociale, l'appréhension claire de ce concept était vitale. En effet, on ne peut faire ouvre consciente de responsabilisation si les composantes de cette action restent inconnues, si la catégorie de jeunes contrevenants qui peut y être davantage sensible n'est pas identifiée, ou si un éventail de mesures est appliqué sans savoir si elles aident vraiment l'adolescent étiqueté délinquant à redevenir un citoyen actif et valorisé dans ses rôles sociaux.

Au plan logistique, une difficulté particulière a été générée par le fait de travailler sur une clientèle assujettie à une loi nouvelle. En effet, des lenteurs et des rythmes inégaux d'entrée de cas - imputables notamment au rodage insuffisant du système judiciaire — ont créé des 
problèmes de planification et d'utilisation rationnelle du personnel intervieweur. Dans la Loi sur les jeunes contrevenants, la porte d'entrée des cas de délinquance cessait d'être le bureau du Directeur de la protection de la jeunesse (dorénavant appelé directeur provincial, en matière de jeunes contrevenants) et devenait le bureau du Substitut du procureur général (S.P.G.). Ce filtrage était intéressant car le S.P.G. établissant la suffisance de preuve dans les dénonciations déposées par les policiers, seuls les cas reconnus de délinquance étaient acheminés au directeur provincial et, conséquemment, à notre programme d'intervention et de recherche. Cependant, le manque de préparation de certains corps policiers, de procureurs de la Couronne et du système judiciaire en général a causé des lenteurs telles que certains sujets arrivaient parfois dans le programme alors que le délai de prescription du délit était dépassé, ou qu'il se passait quelques semaines sans entrée de cas nouveaux alors qu'une avalanche de nouveaux dossiers suivait ce genre de périodes sèches.

Ce type de situations, en période d'ajustement à l'implantation d'une nouvelle loi, a obligé à des efforts constants afin que la charge de travail n'empêche jamais le respect des délais issus de notre devis: cela était particulièrement important pour les périodes entre la fin de l'intervention et les deux moments de mesure APRÈS. Le respect de ces délais était d'autant plus important que la stimulation de l'intérêt et de la motivation des jeunes était un défi constant. II n'était guère difficile d'obtenir du jeune sa collaboration à la mesure AVANT $\left(0_{1}\right)$, mais les deux mesures APRÈS $\left(0_{2}\right.$ et $\left.0_{3}\right)$ survenant deux et six mois après la FIN du programme d'intervention individualisée, le risque de mortalité expérimentale était fort. Malgré tout, près de $70 \%$ de la population totale escomptée a subi l'ensemble du programme d'intervention et du processus d'évaluation.

Nonobstant ces difficultés, l'étude évaluative s'est bien déroulée et ce, notamment en raison de l'appui reçu des praticiens sociaux chargés du programme d'intervention. Ce sont eux qui avaient, au terme dudit programme, à administrer le questionnaire DF (données factuelles) et leur collaboration a été précieuse à ce niveau. Mais la simple expression de leur adhésion à la démarche évaluative en cours constituait un appui non négligeable dans le cadre d'une étude qui s'étend sur près de trois ans, et dans la perspective d'un transfert éventuel des conclusions de l'évaluation dans la pratique sociale en délinquance.

Cet espoir de retombées sur l'intervention psychosociale auprès des jeunes contrevenants est alimenté par la production de premiers ensembles de données. C'est ainsi que des informations descriptives consistantes sont déjà disponibles en regard des différentes mesures de 
responsabilisation applicables, que l'ouverture des milieux communautaires environnant les jeunes peut être documentée, que des données sont systématisées sur le profil personnel et social des jeunes contrevenants des territoires concernés et que ressort encore une fois l'utilité pour la pratique de certains instruments indicateurs ou prédictifs de délinquance.

L'utilité de cette recherche apparaît donc déjà et elle se confirmera davantage lors de la production du rapport final, en 1987.

\section{Notes et références}

1 La réalisation de la recherche a d'abord été rendue possible la première année par deux subventions initiales du Ministère de la santé et des services sociaux, totalisant $\$ 93622$. Le Centre de services sociaux de Québec a supporté une large part des coûts sous forme d'équipements techniques et de prêt de personnel. La même contribution du C.S.S.Q. s'est maintenue pour la deuxième année alors que le Conseil québécois de la recherche sociale $(\$ 40500)$ et le Fonds Richelieu (\$21600) ont apporté, pour cette seconde étape, le support financier direct.

2. Solliciteur général du Canada, La Loi sur les jeunes contrevenants: points saillants, Ottawa, 1982, pp. 1-2.

3 Bruneau, M. et M.-J. Linteau, Description d'un modèle d'intervention minimale appliqué sur la Rive-Sud de Québec, Québec, Centre de services sociaux de Québec, mai 1983.

${ }^{4}$ Charbonneau, J.-P. et al., Rapport de la Commission parlementaire spéciale sur la protection de la jeunesse, Québec, Direction générale des publications gouvernementales du Ministère des communications, 1982.

5 SZABO, D., Criminologie. Montréal, PUM, 1980.

6 SZABO, D., Ibid.

7 Glasser, W., La "Reality Therapy", nouvelle approche thérapeutique par le réel, Paris, Épi, 1971.

8 G. Malcuit, L. Granger et A. Larocque, Les thérapies behaviorales, Québec, PUL, 1972.

9 RECKLESS, W.C., "A new theory of delinquency and crime", Federal Probation, vol. 25, no 1, $1961: 42-46$.

10 LeAHY, J., Le concept de soi des jeunes filles délinquantes, thèse de doctorat en psychologie, Université de Montréal, département de psychologie. 1974.

11 JENSEN, G.F., "Delinquency and adolescent self emotions: a study of the personal relevance of infraction ", Social Problems, vol. 20, n० 1, 1972: 84-103.

12 MORRIS, A., " Diversion of juvenile offenders from the criminal justice system ", dans: Normand TUTr (éd.), Alternative Strategies for Coping with Crime, London, Blackwell/and Robertson, 1978 : 45-63. 
13 Notre territoire de recherche s'est étendu à seize municipalités de la RiveSud de Québec faisant partie des municipalités régionales de comté (M.R.C.) Desjardins et Chutes-de-la-Chaudière, et cinq municipalités de la Rive-Nord : Sainte-Foy, Val-Bélair, Sillery, Cap-Rouge et Ancienne-Lorette.

14 Glasser, W., op. cit.

15 Cusson, M., Le contrôle social du crime, Paris, PUF, 1983.

16 Laflamme, M., La conséquence à l'acte: du principe répressif au principe éducatif ou thérapeutique, document de travail, Direction des services professionnels, Centre de services sociaux de Québec, février 1983.

17 Malcuit, G., l. Granger et A. Larocque, op. cit.

18 Glasser, W., op. cit.

19 Cusson, M., op. cit., pp. 114 et 115.

20 Cusson, M., Ibid., p. 115.

21 BLAIN-LAMBERT, Louise : Réhabilitation du délinquant : amorce d'un processus de prise en charge de ses délinquants par une communauté, mémoire de maîtrise présenté au Département de psychologie de l'Université de Montréal, octobre 1975.

22 Le 1er avril 1984 correspond à la date d'entrée en vigueur de la Loi sur les jeunes contrevenants. La période de référence devait durer douze mois mais fut étendue à quinze mois afin de recueillir le nombre de sujets escomptés pour constituer notre échantillon (350).

23 Fréchette, M., "Portrait de la délinquance", Les cahiers de l'école de criminologie, Université de Montréal, 1980.

${ }^{24}$ Gough, H.G., California Psychological Inventory, Palo Alto, Consulting Psychologists Press, 1960.

25 Gough, H.G., Ibid.

26 Institut de recherches psychologiques, IPAT - échelle A., Ottawa, 1962.

${ }^{27}$ JeSness, C., Manual for the Jesness Inventory, Palo Alto, Consulting Psychologists Press, 1962.

28 Bruneau, M., M. Laflamme et M.-J. Linteau, Échelle données de vie (DV), Québec, texte non publié, 1984.

29 Bruneau, M., M. Laflamme et M.-J. Linteau, Échelle données factuelles (DF), Québec, texte non publié, 1984.

${ }^{30} 0_{1}, 0_{2}, 0_{3}$ représentent des temps d'observation; " $X$ » représente l'application du programme de responsabilisation.

31 Il est important de repréciser que cette étude d'évaluation est en cours et que les résultats seront publiés à l'hiver 1987. 